\title{
A temperatura e umidade na degradação de fipronil em dois solos de Mato Grosso do Sul
}

\author{
Temperature and moisture on fipronil degradation in two soils of Mato Grosso do Sul State, \\ Brazil
}

\author{
Rômulo Penna Scorza Júnior ${ }^{r^{*}}$ André Andrade Franco ${ }^{\mathrm{I}}$
}

\section{RESUMO}

Este trabalho teve como objetivo avaliar a influência da temperatura e umidade na degradação de fipronil, em condições de laboratório, em dois solos de Mato Grosso do Sul. Os solos $(0-30$ e $50-70 \mathrm{~cm})$ foram incubados nas temperaturas de 30 e $40^{\circ} \mathrm{C}$ e umidades de 50 e $80 \%$ da capacidade de campo. Amostras para análise dos resíduos de fipronil foram retiradas aos $0,3,10,17,24,34$ e 44 dias, sendo os dados das quantidades remanescentes em função do tempo ajustados aos modelos de cinética de primeira ordem e bifásico. O aumento da umidade e temperatura favoreceu a degradação do fipronil em ambos os solos. O modelo de cinética de primeira ordem mostrou-se superior ao bifásico para descrever a degradação do fipronil com valores de meia-vida entre 19 e 74 dias.

Palavras-chave: agrotóxico, persistência, meia-vida.

\section{ABSTRACT}

This research had the aim to evaluate the influence of temperature and moisture on fipronil degradation under laboratory conditions of two soils at Mato Grosso do Sul State. Soil samples $(0-30$ and $50-70 \mathrm{~cm})$ were incubated at temperatures of 30 and $40^{\circ} \mathrm{C}$ and soil moistures of 50 and $80 \%$ field capacity. Soil samples for fipronil residue analysis were collected at 0 , $3,10,17,24,34$, and 44 days after application and remaining amount data as a function of time were fitted to first-order kinetic and biphasic models. Temperature and soil moisture increase did improve fipronil degradation for both soils. First-order kinetics model did show a better performance than biphasic model to describe fipronil degradation with half-life values between 19 and 74 days.

Key words: pesticide, persistence, half-life.

\section{INTRODUÇÃO}

A avaliação da periculosidade ambiental de um agrotóxico é de fundamental importância para minimizar possíveis riscos de contaminação dos recursos naturais. Essa avaliação requer informações sobre seu comportamento ambiental como a lixiviação, sorção e degradação (IBAMA, 2010). A degradação tem como função principal a atenuação dos níveis de resíduos do agrotóxico no solo, caracterizando seu grau de persistência, e pode ocorrer de forma biótica e/ou abiótica. Assim, fatores como umidade, temperatura e tipo de solo podem influenciar em sua degradação (WANG et al., 2010). De maneira geral, solos mais úmidos e com temperaturas mais elevadas tendem a aumentar a taxa de degradação do agrotóxico, diminuindo sua persistência (CASTILLO \& TORSTENSSON, 2007; MARTINEZ et al., 2008).

A descrição da cinética de degradação do agrotóxico no solo por meio de modelos matemáticos é importante para estimar seu valor de meia-vida $\left(\mathrm{TD}_{50}\right)$ e sua taxa de degradação. No Brasil, o valor de $\mathrm{TD}_{50}$ é utilizado para classificar os agrotóxicos com relação a sua periculosidade ambiental (IBAMA, 2010). Estimativas de $\mathrm{TD}_{50}$ devem ser feitas de forma bastante criteriosa e fundamentada, o que exige a escolha adequada do modelo matemático para descrever a cinética de

'Laboratório de Análise de Resíduos de Pesticidas, Embrapa Agropecuária Oeste, CP 449, 79804-970, Dourados, MS, Brasil. E-mail: romulo.scorza@embrapa.br.*Autor para correspondência. 
degradação (ALDOWORTH \& JACKSON, 2008). Diversos modelos matemáticos têm sido utilizados para descrever a cinética de degradação de agrotóxicos nos solos (GUSTAFSON \& HOLDEN, 1990; ALDOWORTH \& JACKSON, 2008), sendo o de cinética de primeira ordem o mais utilizado.

Diante da expansão da cultura da canade-açúcar em Mato Grosso do Sul, é urgente a necessidade de informações sobre a persistência de agrotóxicos utilizados nessa cultura e nas condições edafoclimáticas locais. O inseticida fipronil é utilizado no controle de pragas na cultura da cana-deaçúcar e sua aplicação pode ocorrer diretamente no solo ou de forma aérea (AGROFIT, 2003). Estudos sobre a degradação de fipronil em solos brasileiros foram realizados em Pernambuco, onde as condições edafoclimáticas são diferentes de Mato Grosso do Sul, como, por exemplo, o inverno úmido e o verão seco (MASUTTI \& MERMUT, 2007). Este trabalho teve como objetivo avaliar a influência da temperatura e umidade na degradação de fipronil, em condições de laboratório, em dois solos com texturas contrastantes em regiões produtoras de cana-de-açúcar, em Mato Grosso do Sul.

\section{MATERIAL E MÉTODOS}

Os solos foram coletados no campo experimental da Embrapa Agropecuária Oeste, em Dourados, MS (22॰19’29” LS; 5448'51” LW), em julho de 2011, e em uma área com pastagem, em Deodápolis, MS (22॰14’46” LS; 549'22" LW), em novembro de 2011 . Em Dourados, o solo é classificado como Latossolo Vermelho Distroférrico típico (LVdf) de textura argilosa e, em Deodápolis, como Neossolo Quartzarênico Órtico típico (RQo) (SANTOS et al., 2006). Em cada coleta, oito subamostras foram retiradas ao acaso nas profundidades de $0-30$ e $50-70 \mathrm{~cm}$, utilizandose trado holandês. As subamostras foram misturadas e homogeneizadas para constituírem uma amostra composta de cada local, foram secas ao ar e na sombra, trituradas em moinho e peneiradas em malha de $2 \mathrm{~mm}$. Posteriormente, procedeu-se à análise de resíduos de fipronil nessas amostras compostas, nas quais não foi identificada sua presença. As análises físico-químicas para caracterização dos solos foram realizadas conforme CLAESSEN (1997). O LVdf apresentou valores de matéria orgânica (g $\left.\mathrm{kg}^{-1}\right)$, areia $\left(\mathrm{g} \mathrm{kg}^{-1}\right)$, argila $\left(\mathrm{g} \mathrm{kg}^{-1}\right)$ e $\mathrm{pH}\left(\mathrm{CaCl}_{2}\right)$, respectivamente, iguais a 34,134, 739 e 5,1 para $0-30 \mathrm{~cm}$ e $23,117,772$ e 5,3 para $50-70 \mathrm{~cm}$ de profundidade. Já para o RQo, esses valores foram de 9, 917, 72 e 4,2 para 0-30cm e 7,901, 89 e 4,3 para $50-70 \mathrm{~cm}$ de profundidade.

Para incubação, $50 \mathrm{~g}$ de solo seco foram pesados e adicionados em béqueres com posterior contaminação de $2,8 \mu \mathrm{g}$ de fipronil $\mathrm{g}^{-1}$ de solo, que corresponde a 4 vezes a dose recomendada para controle da broca-da-cana, garantindo que, após degradação de $70 \%$ da dose aplicada, os resíduos no solo fossem superiores ao limite de quantificação do método analítico. A umidade em cada amostra, correspondente a 50 e $80 \%$ da capacidade de campo (CC), foi controlada diariamente com a adição de água ultrapura e com auxílio de uma balança até atingir o peso desejado em cada béquer. A determinação da umidade volumétrica correspondente à $\mathrm{CC}$, para ambos os solos, foi baseada na curva de retenção de água do solo, considerando pressão de 0,1 atm. As amostras foram incubadas em incubadoras tipo BOD, com condições controladas de temperaturas de 30 e $40^{\circ} \mathrm{C}$, na ausência de luz. Os experimentos foram conduzidos por 44 dias, em triplicata, sendo as amostras retiradas aos $0,3,10,17,24,34$ e 44 dias após aplicação. Utilizou-se um delineamento inteiramente casualizado em esquema fatorial triplo, em que os fatores foram solo, temperatura e umidade.

A extração e quantificação dos resíduos de fipronil no solo foram baseadas na metodologia de HADJMOHAMMADI et al. (2006). Para extração, em cada amostra de $50 \mathrm{~g}$ de solo, foram adicionados $100 \mathrm{~mL}$ de metanol grau HPLC. Em seguida, cada amostra foi mantida em mesa agitadora a 216rpm e $25^{\circ} \mathrm{C}$ por duas horas. Após agitação, as amostras foram colocadas em repouso por uma hora e, em seguida, foram transferidos $50 \mathrm{~mL}$ do sobrenadante para balão de fundo redondo que foi evaporado até a secura. Os resíduos de fipronil presentes no balão foram elevados para $2 \mathrm{~mL}$ em acetonitrila grau HPLC e centrifugados a $20^{\circ} \mathrm{C}$ com rotação de 2500 rpm por 30 minutos. Posteriormente, as amostras foram filtradas usando filtro de seringa em celulose regenerada de $0,22 \mu \mathrm{m}$ e armazenadas a $-20^{\circ} \mathrm{C}$. A identificação e quantificação dos resíduos de fipronil foram realizadas utilizando-se HPLC-DAD (modelo Varian LC-920), operando a $220 \mathrm{~nm}$. Para separação, utilizou-se pré- 
coluna e coluna C-18 Polaris ( $250 \mathrm{mmx} 4,6 \mathrm{~mm} \times 5 \mu \mathrm{m})$, com temperatura do forno de $35^{\circ} \mathrm{C}$ e em sistema de gradiente com: $50 \%$ acetonitrila e $50 \%$ água ultrapura $\mathrm{v} / \mathrm{v}$ de $0-10 \mathrm{~min}, 100 \%$ acetonitrila de 10 a 21 minutos e $50 \%$ acetonitrila e $50 \%$ água ultrapura de 21 a 32 minutos, com fluxo de $1 \mathrm{~mL}$ minuto ${ }^{-1}$. O tempo de retenção do fipronil nessas condições foi de 6 minutos e 11 segundos. Os testes de eficiência do método analítico indicaram recuperação de fipronil entre 94 e $104 \%$. O limite de quantificação foi de $0,8 \mu \mathrm{g} \mathrm{g}^{-1}$ de solo.

Os valores das quantidades remanescentes de fipronil, aos 44 dias após aplicação, foram submetidos à análise de variância e as médias comparadas pelo teste t a $5 \%$, utilizando o programa R (R DEVELOPMENT CORE TEAM, 2012). Em seguida, as quantidades remanescentes de fipronil em função do tempo, para cada tipo de solo, profundidade, temperatura e umidade foram ajustadas aos modelos de cinética de primeira ordem e ao bifásico de GUSTAFSON \& HOLDEN (1990). Para tal, utilizouse o programa SigmaPlot ${ }^{\circledR} 12.0$ pelo método do ajuste de regressão não linear. $\mathrm{O}$ modelo de cinética de primeira ordem é dado por $\mathrm{C}=\mathrm{C}_{0} \cdot \mathrm{e}^{-\mathrm{kt}}$ em que $\mathrm{C}$ é a quantidade remanescente de fipronil no solo (\% da dose aplicada), $\mathrm{C}_{0}$ é a quantidade inicial aplicada (\%), $\mathrm{k}$ é a taxa de degradação $\left(\right.$ dia $\left.^{-1}\right)$ e $\mathrm{t}$ é o tempo (dias). $\mathrm{O}$ modelo bifásico é dado por $\mathrm{C}=\mathrm{C}_{0} \cdot(1+\beta \cdot \mathrm{t})^{-\alpha}$ em que $\alpha$ e $\beta$ ( $\left(\mathrm{dia}^{-1}\right)$ são parâmetros. A qualidade do ajuste dos dados observados aos dois modelos foi feita com base na análise visual e Erro $\chi^{2}$ (FOCUS, 2006). Quanto menor o valor do Erro $\chi^{2}$, considerando que este tenha passado pelo teste chi-quadrado $\left(\chi^{2}\right)$, mais robusto é o modelo para descrever os dados observados. Os valores de $\mathrm{TD}_{50}$ do fipronil, nas diferentes condições estudadas, foram estimados com base em $\mathrm{TD}_{50}=\frac{0,693}{\mathrm{k}}$ para o modelo de cinética de primeira ordem e em $\mathrm{TD}_{50}=\frac{1}{\beta} \cdot\left(2^{\frac{1}{\alpha}}-1\right)$ para o modelo bifásico.

\section{RESULTADOS E DISCUSSÃO}

Para a profundidade de $0-30 \mathrm{~cm}$, observouse interação significativa $(\mathrm{P}<0,05)$ entre solo, umidade e temperatura na degradação do fipronil. Devido à natureza contrastante dos solos estudados, os resultados de cada solo foram apresentados separadamente (Tabela 1). Para ambas as temperaturas e solos, o aumento da umidade de 50 para $80 \%$ da CC favoreceu significativamente $(\mathrm{P}<0,05)$ o aumento da degradação do fipronil. Já para ambas as umidades e solos, a elevação da temperatura de 30 para $40^{\circ} \mathrm{C}$ também favoreceu, de forma significativa $(\mathrm{P}<0,05)$, o aumento da degradação do fipronil, com exceção para a umidade correspondente a $80 \%$ da CC no solo arenoso de Deodápolis. Para a profundidade de 50$70 \mathrm{~cm}$, a interação entre os fatores solo, temperatura e umidade não foi significativa $(\mathrm{P}>0,05)$. No entanto, observou-se efeito significativo $(\mathrm{P}<0,05)$ desses fatores quando combinados dois a dois (Tabela 2). De maneira geral, o aumento de temperatura e umidade favoreceu a degradação do fipronil, em ambos os solos. A degradação do fipronil foi mais rápida no LVdf, quando comparada ao RQo, em ambas as temperaturas e umidades avaliadas. A degradação de fipronil em solos é influenciada por fatores ambientais como, por exemplo, umidade, temperatura, $\mathrm{pH}$, tipo de solo e atividade microbiana (YING \& KOOKANA, 2002; MASUTTI \& MERMUT, 2007; KUMAR et al., 2012). MASUTTI \& MERMUT (2007) concluíram que a degradação do fipronil em solos incubados em laboratório e provenientes de área com canade-açúcar, em Pernambuco, foi predominantemente biótica. Assim, os microrganismos do solo têm papel

Tabela 1 - Quantidades remanescentes (\%) de fipronil nos dois solos, temperaturas e umidades avaliadas na profundidade de $0-30 \mathrm{~cm}$.

\begin{tabular}{|c|c|c|c|c|}
\hline \multirow[t]{2}{*}{ Umidade (\% CC) } & \multicolumn{4}{|c|}{-----------------------------Dourados---------------------------- } \\
\hline & 30 & 40 & 30 & 40 \\
\hline 50 & $83,07 \mathrm{aA}$ & $70,01 \mathrm{bA}$ & $85,34 \mathrm{aA}$ & $61,89 \mathrm{bA}$ \\
\hline 80 & $45,13 \mathrm{aB}$ & $34,47 \mathrm{bB}$ & $54,02 \mathrm{aB}$ & $53,55 \mathrm{aB}$ \\
\hline
\end{tabular}

Médias seguidas por letras iguais, minúsculas nas linhas e maiúsculas nas colunas, dentro de cada local, não diferem entre si pelo teste $\mathrm{t}$ a $5 \%$. Comparação entre umidades nas colunas e entre temperaturas nas linhas, para cada local. $\mathrm{CC}=$ capacidade de campo. $\mathrm{CV}=19 \%$. 
Tabela 2 - Quantidades remanescentes (\%) de fipronil nos dois solos, temperaturas e umidades avaliadas na profundidade de $50-70 \mathrm{~cm}$

\begin{tabular}{lcc}
\hline \multirow{2}{*}{ Solo } & \multicolumn{2}{c}{---------Temperatura $\left({ }^{\circ} \mathrm{C}\right)$------------ } \\
& $51,76 \mathrm{aB}$ & 40 \\
\hline Dourados & $96,77 \mathrm{aA}$ & $26,11 \mathrm{bB}$ \\
Deodápolis & 50 & $78,04 \mathrm{bA}$ \\
Solo & $34,47 \mathrm{bB}$ & 80 \\
& $92,26 \mathrm{aA}$ & $82,39 \mathrm{aB}$ \\
Dourados & 30 & $40 \mathrm{bA}$ \\
Deodápolis & $76,89 \mathrm{aB}$ & $49,85 \mathrm{bA}$ \\
Umidade $(\% \mathrm{CC})$ & $71,64 \mathrm{aA}$ & $54,31 \mathrm{bB}$ \\
\hline
\end{tabular}

Médias seguidas por letras iguais, minúsculas nas linhas e maiúsculas nas colunas, não diferem entre si pelo teste t a $5 \%$. $\mathrm{CC}=$ capacidade de campo. $\mathrm{CV}=18 \%$.

importante na degradação do fipronil que, por sua vez, são influenciados pelas variações de temperatura e umidade que regulam sua atividade. $\mathrm{O}$ aumento de temperatura e umidade do solo dentro dos limites ótimos para os microrganismos acelera a degradação de agrotóxicos (CASTILLO \& TORSTENSSON, 2007; MARTINEZ et al., 2008). Os valores do erro $\chi^{2}$ para o ajuste do modelo de cinética de primeira ordem às concentrações remanescentes de fipronil em função do tempo foram, em sua maioria, inferiores àqueles observados para o modelo bifásico, considerando os dois solos e as diferentes condições de temperatura e umidade (Tabelas 3 e 4 ). Isso mostra a superioridade do modelo de cinética de primeira ordem para descrever a degradação do fipronil nas condições avaliadas. No entanto, essa superioridade mostrou-se pequena, devido às diferenças na qualidade do ajuste dos dois modelos aos dados observados não serem visualmente discrepantes como, por exemplo, mostrado para a degradação no LVdf a $40^{\circ} \mathrm{C}$ (Figura 1). O modelo de cinética de primeira ordem é utilizado para descrever a degradação do tipo monofásica de agrotóxicos em solos. Não foi observado comportamento de degradação do tipo bifásica para o fipronil nos solos e condições avaliadas (Figura 1). Degradação do tipo monofásica para o fipronil também foi observada por SHUAI et al. (2012). Diferentemente, MASUTTI \& MERMUT (2007) observaram degradação do tipo

Tabela 3 - Parâmetros do modelo de degradação de cinética de primeira ordem para degradação de fipronil no Latossolo Vermelho distrófico (LVdf) e Neossolo Quartzarênico Órtico típico (RQo) nas duas temperaturas e umidades.

\begin{tabular}{|c|c|c|c|c|c|c|c|}
\hline Solo & Temp. $\left({ }^{\circ} \mathrm{C}\right)$ & Prof. (cm) & Umidade $(\% \mathrm{CC})$ & Erro $\chi^{2}(\%)$ & $\mathrm{C}_{0}(\%)$ & $\mathrm{k}\left(\mathrm{dia}^{-1}\right)$ & $\mathrm{TD}_{50}$ (dias) \\
\hline \multirow{11}{*}{ LVdf } & \multirow{5}{*}{30} & \multirow{2}{*}{$0-30$} & 50 & 1,4 & $100 \pm 1^{*}$ & $0,0038 \pm 0,0004^{*}$ & - \\
\hline & & & 80 & 2,5 & $98 \pm 1^{*}$ & $0,0182 \pm 0,0008^{*}$ & 38 \\
\hline & & & & & & & \\
\hline & & \multirow{2}{*}{$50-70$} & 50 & 2,4 & $100 \pm 1^{*}$ & $0,0149 \pm 0,0007^{*}$ & 47 \\
\hline & & & 80 & 3,7 & $102 \pm 2^{*}$ & $0,0132 \pm 0,0009^{*}$ & 53 \\
\hline & \multirow{6}{*}{40} & & & & & & \\
\hline & & \multirow{2}{*}{$0-30$} & 50 & 2,2 & $101 \pm 1^{*}$ & $0,0081 \pm 0,0005^{*}$ & - \\
\hline & & & 80 & 2,3 & $97 \pm 1^{*}$ & $0,0254 \pm 0,0007^{*}$ & 28 \\
\hline & & & & & & & \\
\hline & & \multirow{2}{*}{$50-70$} & 50 & 1,4 & $100 \pm 1^{*}$ & $0,0360 \pm 0,0011^{*}$ & 19 \\
\hline & & & 80 & 2,3 & $101 \pm 1^{*}$ & $0,0239 \pm 0,0010^{*}$ & 29 \\
\hline \multirow{11}{*}{ RQo } & \multirow{6}{*}{30} & \multirow{2}{*}{$0-30$} & 50 & 1,2 & $100 \pm 1^{*}$ & $0,0038 \pm 0,0005^{*}$ & - \\
\hline & & & 80 & 2,1 & $102 \pm 1^{*}$ & $0,0149 \pm 0,0007^{*}$ & 46 \\
\hline & & & & & & & \\
\hline & & \multirow{2}{*}{$50-70$} & 50 & - & - & - & - \\
\hline & & & 80 & 1,0 & $99 \pm 1^{*}$ & $0,0026 \pm 0,0003^{*}$ & - \\
\hline & & & & & & & \\
\hline & \multirow{5}{*}{40} & \multirow{2}{*}{$0-30$} & 50 & 2,6 & $100 \pm 1^{*}$ & $0,0094 \pm 0,0007^{*}$ & 74 \\
\hline & & & 80 & 3,2 & $98 \pm 2^{*}$ & $0,0163 \pm 0,0010^{*}$ & 43 \\
\hline & & & & & & & \\
\hline & & \multirow{2}{*}{$50-70$} & 50 & 1,8 & $99 \pm 1^{*}$ & $0,0039 \pm 0,0004^{*}$ & - \\
\hline & & & 80 & 0,8 & $101 \pm 1^{*}$ & $0,0064 \pm 0,0004^{*}$ & - \\
\hline
\end{tabular}

${ }^{*}$ Significativo pelo teste t a $5 \%$. CC $=$ capacidade de campo. $\mathrm{TD}_{50}=$ meia-vida. 
Tabela 4 - Parâmetros do modelo bifásico para degradação de fipronil no solo Latossolo Vermelho distrófico (LVdf) e Neossolo Quartzarênico Órtico típico (RQo) nas duas temperaturas e umidades.

\begin{tabular}{|c|c|c|c|c|c|c|c|c|}
\hline Solo & Temp. $\left({ }^{\circ} \mathrm{C}\right)$ & Prof. (cm) & Umidade (\%CC) & Erro $\chi^{2}(\%)$ & $\mathrm{C}_{0}(\%)$ & $\alpha(-)$ & $\beta$ (dia) & $\mathrm{TD}_{50}$ (dias) \\
\hline \multirow{11}{*}{ LVdf } & \multirow{5}{*}{30} & \multirow{2}{*}{$0-30$} & 50 & 1,8 & $100 \pm 1^{*}$ & $0,31 \pm 0,56^{\mathrm{ns}}$ & $0,02 \pm 0,04^{\mathrm{ns}}$ & - \\
\hline & & & 80 & 3,2 & $101 \pm 2^{*}$ & $0,77 \pm 0,32^{*}$ & $0,03 \pm 0,02^{\mathrm{ns}}$ & 40 \\
\hline & & & & & & & & \\
\hline & & \multirow{2}{*}{$50-70$} & 50 & 4,7 & $101 \pm 3^{*}$ & $0,51 \pm 0,26^{\mathrm{ns}}$ & $0,05 \pm 0,04^{\mathrm{ns}}$ & 55 \\
\hline & & & 80 & 4,9 & $103 \pm 3^{*}$ & $0,93 \pm 1,14^{\mathrm{ns}}$ & $0,02 \pm 0,03^{\mathrm{ns}}$ & 59 \\
\hline & \multirow{6}{*}{40} & & & & & & & \\
\hline & & \multirow{2}{*}{$0-30$} & 50 & 2,8 & $102 \pm 2^{*}$ & $0,86 \pm 1,26^{\mathrm{ns}}$ & $0,01 \pm 0,02^{\mathrm{ns}}$ & - \\
\hline & & & 80 & 1,8 & $99 \pm 1^{*}$ & $1,63 \pm 0,43^{*}$ & $0,02 \pm 0,01^{*}$ & 26 \\
\hline & & & & & & & & \\
\hline & & \multirow{2}{*}{$50-70$} & 50 & 4,0 & $102 \pm 2^{*}$ & $2,24 \pm 1,15^{\mathrm{ns}}$ & $0,02 \pm 0,01^{\mathrm{ns}}$ & 18 \\
\hline & & & 80 & 4,1 & $102 \pm 2^{*}$ & $1,72 \pm 1,26^{\mathrm{ns}}$ & $0,02 \pm 0,01^{\mathrm{ns}}$ & 28 \\
\hline \multirow{11}{*}{ RQo } & \multirow{5}{*}{30} & \multirow{2}{*}{$0-30$} & 50 & 0,9 & $100 \pm 1^{*}$ & $0,24 \pm 0,29^{\mathrm{ns}}$ & $0,02 \pm 0,04^{\mathrm{ns}}$ & - \\
\hline & & & 80 & 2,3 & $104 \pm 2^{*}$ & $0,74 \pm 0,37^{\mathrm{ns}}$ & $0,03 \pm 0,02^{\mathrm{ns}}$ & 47 \\
\hline & & & & & & & & \\
\hline & & \multirow{2}{*}{$50-70$} & 50 & - & - & - & - & - \\
\hline & & & 80 & 0,8 & $100 \pm 1^{*}$ & $0,17 \pm 0,17^{\mathrm{ns}}$ & $0,02 \pm 0,03^{\mathrm{ns}}$ & - \\
\hline & \multirow{6}{*}{40} & & & & & & & \\
\hline & & \multirow{2}{*}{$0-30$} & 50 & 3,7 & $102 \pm 2^{*}$ & $0,44 \pm 0,34^{\mathrm{ns}}$ & $0,03 \pm 0,04^{\mathrm{ns}}$ & - \\
\hline & & & 80 & 3,2 & $101 \pm 2^{*}$ & $0,56 \pm 0,21^{*}$ & $0,05 \pm 0,03^{\mathrm{ns}}$ & 47 \\
\hline & & & & & & & & \\
\hline & & \multirow{2}{*}{$50-70$} & 50 & 2,1 & $100 \pm 1^{*}$ & $0,29 \pm 0,43^{\mathrm{ns}}$ & $0,02 \pm 0,03^{\mathrm{ns}}$ & - \\
\hline & & & 80 & 1,4 & $102 \pm 1^{*}$ & $0,46 \pm 0,46^{\mathrm{ns}}$ & $0,02 \pm 0,02^{\mathrm{ns}}$ & - \\
\hline
\end{tabular}

${ }^{*}$ Significativo pelo teste $\mathrm{t}$ a $5 \%$; ns = não significativo pelo teste t a $5 \% . \mathrm{CC}=$ capacidade de campo. $\mathrm{TD}_{50}=$ meia-vida.

bifásica para o fipronil em solos, com uma fase inicial lenta, seguida de uma mais acelerada após 90 dias de incubação.

Os valores de $\mathrm{TD}_{50}$, com base no modelo de cinética de primeira ordem, variaram de 19 a 47 dias para o LVdf e de 43 a 74 dias para o RQo. Optou-se por estimar o valor de $\mathrm{TD}_{50}$ apenas para os experimentos em que a quantidade remanescente de fipronil, na última data de amostragem, foi inferior a $50 \%$. A menor persistência do fipronil $\left(\mathrm{TD}_{50}\right.$ igual a 19 dias) foi observada no LVdf para a profundidade de $50-70 \mathrm{~cm}$, temperatura de $40^{\circ} \mathrm{C}$ e umidade de $50 \%$ da CC. Valores de meia-vida para o fipronil em solos incubados em laboratório e estimados com base no modelo de cinética de primeira ordem são bastante variáveis em função das condições estudadas, como, por exemplo, temperatura e umidade do solo, tipo de solo e profundidade. MASUTTI \& MERMUT (2007) relatam valores de meia-vida do fipronil entre 83 e 200 dias para solos superficiais $(0-15 \mathrm{~cm})$, incubados a $25^{\circ} \mathrm{C}$ e entre 55 e $59 \%$ da $\mathrm{CC}$, coletados em uma área com cana-de-açúcar em Pernambuco. YING \& KOOKANA (2002) observaram valores de meia- vida iguais a 68 e 198 dias para solos australianos superficiais $(0-15 \mathrm{~cm})$, incubados a $20^{\circ} \mathrm{C}$ e com umidades de 60 e $15 \%$ da CC, respectivamente. Já SHUAI et al. (2012) relatam valores bem inferiores de meia-vida do fipronil, entre 28 e 34 dias, para solos superficiais $(0-20 \mathrm{~cm})$ do Havaí, incubados a $30^{\circ} \mathrm{C}$ e $75 \%$ da CC.

\section{CONCLUSÃO}

Os aumentos da temperatura e umidade do solo aceleram a degradação do fipronil, sendo esta descrita de forma satisfatória pelo modelo de cinética de primeira ordem. A meia-vida do fipronil variou de 19 a 74 dias nos solos e condições estudadas, classificando-o como agrotóxico de baixa a média persistência.

\section{AGRADECIMENTOS}

À Fundação de Apoio ao Desenvolvimento de Ensino, Ciência e Tecnologia de Mato Grosso do Sul (FUNDECT-MS T.O. 008/09) e ao CNPq (35.0062/2009-9 e 475539/2010-9).

Ciência Rural, v.43, n.7, jul, 2013. 


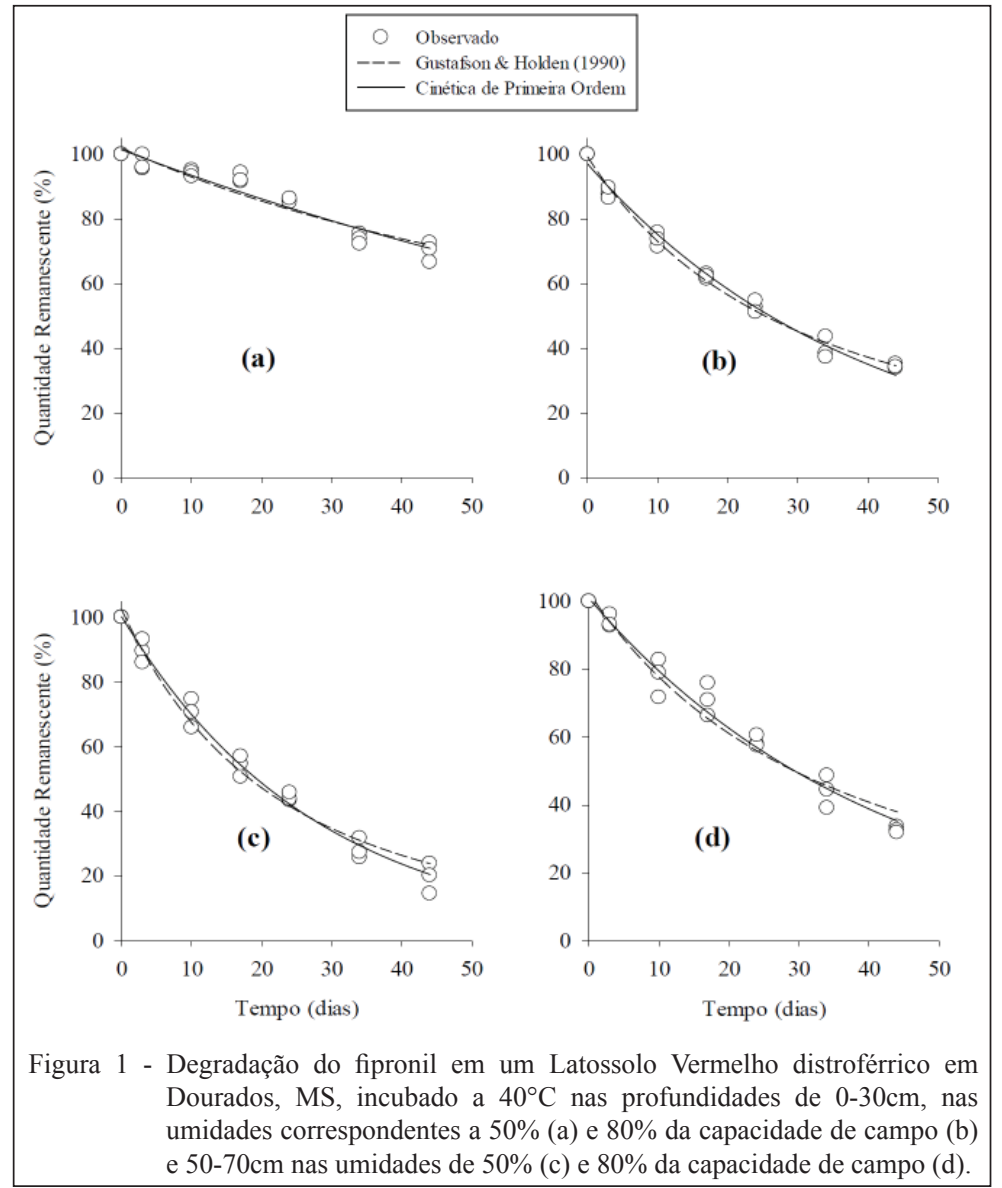

\section{REFERÊNCIAS}

AGROFIT: Sistema de Agrotóxicos Fitossanitários. Brasília: Ministério da Agricultura, Pecuária e Abastecimento, 2003. Disponível em: <http://extranet.agricultura.gov.br/agrofit_cons/ principal_agrofit_cons $>$. Acesso em: 15 ago. 2012.

ALDOWORTH, J.; JACKSON, S.H. Statistical tools for determining appropriate selection of regression models for analysis of environmental fate datasets. Pesticide and Management Science, v.64, n.5, p.536-543, 2008. Disponível em: <http:// onlinelibrary.wiley.com/doi/10.1002/ps.1537/abstract>. Acesso em: 24 jul. 2012. doi: 10.1002/ps. 1537

CASTILLO, M.D.P.; TORSTENSSON, L. Effect of biobed composition, moisture, and temperature on the degradation of pesticides. Journal of Agricultural Food and Chemistry, v.55, n.14, p.5725-5733, 2007. Disponível em: <http://pubs.acs.org/toc/ jafcau/55/14>. Acesso em: 12 jul. 2012. doi: 10.1021/jf0706637.

CLAESSEN, M.E.C. Manual de métodos de análise de solo. Rio de Janeiro: Embrapa-CNPS, 1997. 212p. (Embrapa-CNPS. Documentos, 1).

FOCUS. Guidance document on estimating persistence and degradation kinetics from environmental fate studies on pesticides in EU registration. Brussels: EC, 2006. 434p. Disponível em: $<$ http://focus.jrc.ec.europa.eu/dk/doc.html $>$. Acesso em: 24 jul. 2012.
GUSTAFSON, D.; HOLDEN, L.R. Nonlinear pesticide dissipation in soil: a new model based on spatial variability. Environmental Science and Technology, v.24, n.7, p.1032-1038, 1990. Disponível em: $<$ http://pubs.acs.org/toc/esthag/24/7>. Acesso em: 24 jul. 2012. doi: 10.1021/es00077a013.

HADJMOHAMMADI, M.R. et al. Determination of fipronil residue in soil and water in the rice fields in north of Iran by RPHPLC method. Acta Chimica Slovenica, v.53, n.4, p.517-520, 2006. Disponível em: <http://acta.chem-soc.si/53/graph/acta53(4)-GA.htm>. Acesso em: 16 jul. 2012.

IBAMA. Produtos agrotóxicos e afins comercializados em 2009 no Brasil. Brasília: Ibama, 2010. 84p. Disponível em: $<$ http://www.ibama.gov.br\%2Fphocadownload\%2FQualidade Ambienta1\%2Fprodutos_agrotoxicos_comercializados_brasil 2009.pdf>. Acesso em: 24 jul. 2012.

KUMAR, R. et al. Biodegradation of fipronil by Paracoccus sp. in different types of soils. Bulletin of Environmental Contamination and Toxicology, v.88, n.5, p.781-787, 2012. Disponível em: $<$ http:// www.springer.com/environment/pollution+and+remediation/ journal/128>. Acesso em: 11 jul. 2012. doi:10.1007/s00128-0120578-y.

MARTINEZ, C.O. et al. The effects of moisture and temperature on the degradation of sulfentrazone. Geoderma, v.147, n.1, p.5662, 2008. Disponível em: <http://www.sciencedirect.com/science/ 
journal/00167061/147/1-2>. Acesso em: 12 jul. 2012. doi: 10.1016/j.geoderma.2008.07.005.

MASUTTI, C.S.M.; MERMUT, A.R. Degradation of fipronil under laboratory conditions in a tropical soil from Sirinhaém Pernambuco, Brazil. Journal of Environmental Science and Health Part B, v.42, n.1, p.33-43, 2007. Disponível em:< http:// www.tandfonline.com/toc/lesb20/42/1>. Acesso em: 11 jul. 2012. doi: $10.1080 / 03601230601017981$.

R DEVELOPMENT CORE TEAM. R: a language and environment for statistical computing. Vienna: R Foundation for Statistical Computing, 2012. Disponível em: http://www.Rproject.org/. Acesso em: 24 jul. 2012.

SANTOS, H.G. dos et al. (Ed.). Sistema brasileiro de classificação de solos. Rio de Janeiro: Embrapa Solos, 2006. 306p.
SHUAI, X. et al. Adsorption, transport and degradation of fipronil termiticide in three Hawaii soils. Pest and Management Science, v.68, n.5, p.731-739, 2012. Disponível em: <http://onlinelibrary. wiley.com/doi/10.1002/ps.v68.5/issuetoc $>$. Acesso em: 11 jul. 2012. doi: $10.1002 / \mathrm{ps} .2320$.

WANG, W. et al. Influence of soil factors on the dissipation of a new pyrimidynyloxybenzoic herbicide ZJ0273. Journal of Agricultural and Food and Chemistry, v.58, n.5, p.3062-3067, 2010. Disponível em: <http://pubs.acs.org/toc/jafcau/58/5>. Acesso em: 24 jul. 2012. doi:10.1021/jf904105b.

YING, G.G.; KOOKANA, R. Laboratory and field studies on the degradation of fipronil in a soil. Australian Journal of Soil Research, v.40, n.7, p.1095-1102, 2002. Disponível em:<http:// www.publish.csiro.au/nid/85/issue/581.htm>. Acesso em: 11 jul. 2012. doi: $10.1071 /$ SR02018.

Ciência Rural, v.43, n.7, jul, 2013. 